\title{
Intelligent interaction based on holographic personalized portal
}

\author{
Yadong Huang, Yueting Chai, Yi Liu and Xiang Gu
}

Department of Automation, National Engineering Laboratory for E-Commerce

Technologies, Tsinghua University, Beijing, China

\begin{abstract}
Purpose - The purpose of this paper is to study the architecture of holographic personalized portal, user modeling, commodity modeling and intelligent interaction.

Design/methodology/approach - In this paper, the authors propose crowd-science industrial ecological system based on holographic personalized portal and its interaction. The holographic personality portal is based on holographic enterprises, commodities and consumers, and the personalized portal consists of accurate ontology, reliable supply, intelligent demand and smart cyberspace.

Findings - The personalized portal can realize the information acquisition, characteristic analysis and holographic presentation. Then, the intelligent interaction, e.g. demand decomposition, personalized search, personalized presentation and demand prediction, will be implemented within the personalized portal.

Originality/value - The authors believe that their work on intelligent interaction based on holographic personalized portal, which has been first proposed in this paper, is innovation focusing on the interaction between intelligence and convenience.
\end{abstract}

Keywords E-commerce, User modelling, Holographic information, Intelligent interaction, Personalized portal

Paper type Research paper

\section{Introduction}

With the continuously growing internet technology, e-commerce has been one of the most important channels of transaction. Openness, interconnection, cooperation and sharing will be the basic logic in the future network society. Intelligent network, which is large-scaled, open-styled, self-organized and ecological, consisting of numerous interconnected intelligent subjects, will be the integration of information, physics and consciousness. And the market will become decentralized and disintermediated gradually.

To comply with the trend of network society, this paper proposes a crowd-science industrial ecological system based on the holographic portal and its interaction, takes the block-chain data storage as safeguard, which gathers the information involved in the business process. The holographic personality portal based on holographic enterprises, commodities and consumers. Each participant has his own personalized portal, through which all the interactions are completed.

(C) Yadong Huang, Yueting Chai, Yi Liu and Xiang Gu. Published in the International Journal of Crowd Science. Published by Emerald Publishing Limited. This article is published under the Creative Commons Attribution (CC BY 4.0) licence. Anyone may reproduce, distribute, translate and create derivative works of this article (for both commercial and non-commercial purposes), subject to full attribution to the original publication and authors. The full terms of this licence may be seen at $\mathrm{http} / / /$ creativecommons.org/licences/by/4.0/legalcode

Received 16 August 2017 Revised 27 August 2017 Accepted 28 August 2017 
IJCS

1,2

User information forming the personalized portal can be obtained by various ways, and the physical, knowledge and remind information can be presented thoroughly after analyzing the user's personalized information and behavior, e.g. repeated search, repeated purchase, demand correlation, and commodity attributes co-occurrence. Similarly, the holographic commodity information should be gathered from both the supply side and demand side, and characteristics of commodity should be analyzed. After the user and commodity modeling, the intelligent interaction can be conducted within user portal, demand decomposition, personalized search, personalized presentation and demand prediction.

\section{Previous literature}

\subsection{User preference acquisition}

The consumers pursue the individuation, style and spiritual enjoyment, and the form and content of the consumption are becoming more and more diversified. The repeat purchase rate of some commodities is low, because the preference varies all the time (Koren, 2009). For example, a user who has a red T-shirt may not buy another one, because of the drift of personalized psychology or interest. As a result, it is more and more important to acquire the user preference quickly and accurately.

The acquisition of user preferences is mainly from two perspectives: analysis of the user's online behavior and commodity attributes. At present, there are explicit and implicit methods to obtain user preference (Aggarwal and Yu, 2000). The explicit method obtains the user preference directly through dynamic feedback, interactive and questionnaires (Bruyn et al., 2008); for example, WebWatcher and MyYahoo need user to mark their interesting keywords and projects (Joachims et al., 1997). The implicit method obtains user preference through analyzing the browsing information and selection history (Ying, 2003). It is easy to update the user preference for implicit method because of the low user engagement, but there may be differences between the induced preference and real situation (Claypool et al., 2001).

Google adopts a theme to describe the user's attention to the content of information. Gediminas Adomavicius uses multi-dimensional space model to express the score matrix of collaborative filtering recommendation algorithm (Adomavicius et al., 2005). In addition, there are weighted keyword vectors, case-based representations (Smyth et al., 2002), fine grain model representations and so on.

\subsection{Demand modeling method}

The main methods of user modeling are genetic algorithm and machine learning, e.g. econometric method, system dynamic method, automatic clustering, decision tree and neural network.

Econometric methods are mainly developed from short-term forecasts, while this approach can do nothing on the unstable economic process and the economic phenomenon lacking of normative behavior theory (Williams et al., 2002).

System dynamics method based on feedback theory and computer simulation was proposed by J.W. Forrester first in 1950s, which is used for predicting the medium long-term trends and properties of system behavior. The system dynamics method is capable of predicting neither a particular event nor an action in the future.

Items or users with similar features will be grouped in the clustering system. The probability that the user browses or visits the recommended object belongs to a given class will be used in resource items classification, which constitutes the user preference (Santos and Zárate, 2015). 
The user starts from the root node and is directed to answer a series of questions until a decision tree is formed. Each node of the tree represents a decision point, and once the leaf node is reached, a complete description of user preference is obtained (Lomax and Vadera, 2013).

Artificial neural network method was applied in economic forecasting by Lapedes and Farber in 1987, which can be used in both short-term and medium long-term economic forecasting. The input hypothesis of user preference is studied and the weights of the network connections are adjusted until all the nodes reach stability. Then the content of the active node in the output layer represents the user preference.

\subsection{Psychology in recommendation}

The traditional recommender systems only consider the material demand in the analysis of user preferences and ignore the psychological factors. As a result, the individual psychological needs of users cannot be obtained by the recommend system, which does not produce good recommendation effects. Therefore, it is necessary to take the psychological needs of users into account in the personalized recommendation system, which may make up for the shortcomings of traditional methods in mining user preferences.

Ngip Khean Chuan adopted Kansei engineering in analyzing the psychological needs of users on the internet and used interactive way to recommend the proper commodity to the user (Chuan et al., 2013). Amaud De Bruyn built a psychological model based on the simple demographic information, the usage of commodity and the explicit consumption preferences, which helps in purchasing decisions (Bruyn et al., 2008).

\section{The system architecture}

\subsection{Overall architecture}

To explore the evolution of the market, a macro-level architecture should be established first to characterize the crowd-science industrial ecological as shown in Figure 1.

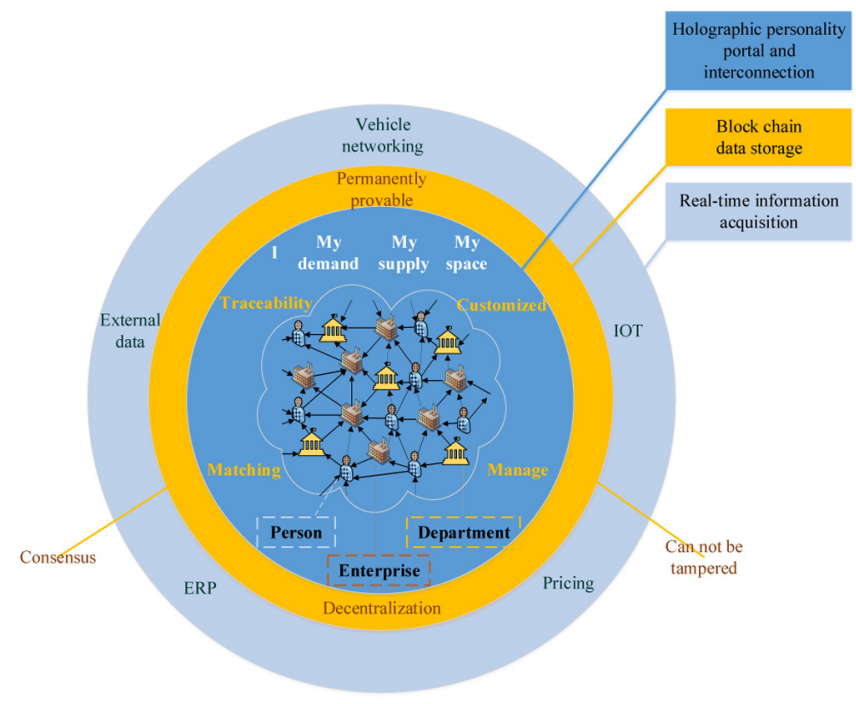

Figure 1.

The system architecture of crowdscience industrial ecological
Holographic personalized portal 
IJCS

1,2

The crowd-science industrial-ecological system based on the holographic personality portal and its interaction, takes the block-chain data storage as safeguard, which gathers the information involved in the business process, e.g. enterprise resource planning (ERP) information, internet of things (IOT) information, pricing information, vehicle information and external data.

The holographic personality portal based on holographic enterprises, commodities and consumers, the personalized portal of enterprise and consumer are the carriers, which achieves the precise interconnection and intelligent transaction between any two portals. Within the personalized portal, the owner can complete proactive and personalized consumption, direct and centralized circulation, decentralized and intelligent production. Finally, the self-organized, open-end and ecological industrial operation system is formed.

The advantages of block chain can be expressed as follows:

- An indelible record of public books can ensure all the information within the personalized portal credible.

- The data can be checked by the trusted third-party department, e.g. testing institution and regulators.

- Alliance chain-consensus mechanism provides admittance service, which meets compliance requirements.

- Automatic data storage of IOT and the certificate of deposit can be audited.

\subsection{Personalized portal}

Nowadays, most of the trading platforms act as mediations, which is against the tendency of disintermediation. The platform will gain the data generated by all of its participants, which will result in harmful monopolization. However, user of personalized portal can complete the demand recognition, supply release, matching, transaction and credit evaluation within his own personalized portal.

Each participant has his own personalized portal, through which all the interactions are completed as shown in Figure 2. The numerous portals are interconnected through four channels, accurate ontology, reliable supply, intelligent demand and smart cyberspace.

Figure 2.

The constitute of personalized portal

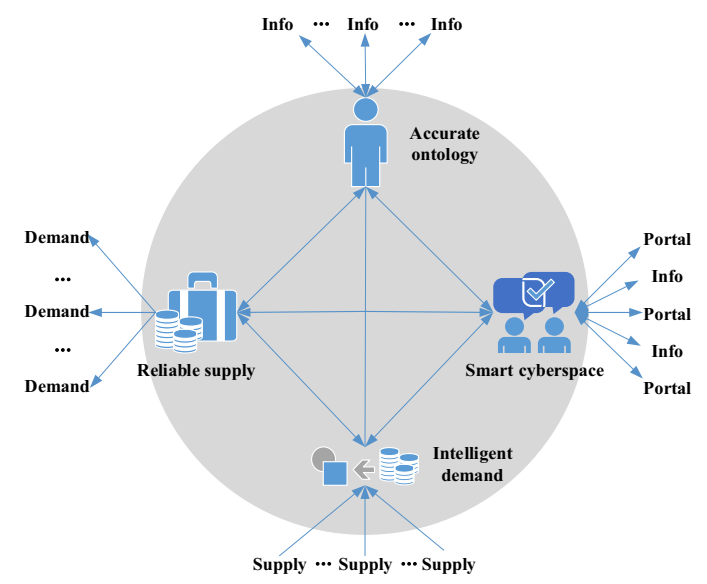


Accurate ontology consists of information and behavior from the realistic world, and it is the counterpart to reflect realistic individual or organization. It can provide the user basic information, 3D model and knowledge graph. Reliable supply provides supply release, history, preference and status of the subject thoroughly through words, images, visual reality (VR), augmented reality (AR), real-time visuals (RTV) and real-time interaction. Similarly, demand proposing, history, preference and status of the subject can be presented thoroughly through words, images, VR, AR, RTV and real-time interaction. The personalized demand information, based on the user preference, basic information and personalized portal instant demand information, is recognized by the demand-recognition system. Then the intelligent matching system will provide a configurable and holographic supply chain to the user. Smart cyberspace collects and arranges the information automatically from the owner and the ones who have interaction with him, based on the social network of user-centered forms with the weights and relation updating in real time. The smart cyberspace, as a way to know the world, will solve the isolated islands of information, which may improve the operation efficiency of whole society.

\section{User modeling}

\subsection{Data source}

User information, forming the personalized portal, can be obtained from the following ways:

- User can import his own information into the system, which is the most straightforward way, and it will help with the cold start situation.

- Any action from the user will be analyzed inside and outside the personalized portal, e.g. search, click, brows, consult and transaction. Finally, the logical information world and the actual physical world can be syncretized, which will increase the accuracy of demand identification.

- The real-time information associated with the user can be gathered by IOT and wearable devices, which ensures the synchronization of user information and this mechanism is crucial for the instant demand.

- Scattered pieces of information studied intelligently from the social network can contribute to the potential demand based on his similar users, and the accuracy of recommendation may be improved.

In addition, user data should be synchronized on different devices, including mobile, laptop, desktop, etc.

\subsection{Characteristic analysis}

4.2.1 Repeated search. Repeated searches refer to the user's submits of the same keywords at different times. It is more important to study the situation in which the user clicks the different results in the repeated searches than clicking the same results. As users' demands vary widely, each user may choose different commodities from the same search results. So the quality of search results can be improved by taking the user's personalized information into account.

In a search session, user may sequentially submit multiple filter criteria $\left(F_{0}, F_{1}, \ldots, F_{k}\right)$. For the current filter criterion $F_{k}$, its related short-term history data consist of history filters criteria $H_{F}$ and history clicks $H_{C}$, where $H_{F}=\left(F_{0}, F_{1}, \ldots, F_{k-1}\right)$ and $H_{C}=\left(C_{0}, C_{1}, \ldots, C_{k-1}\right)$ means the submitted filter criteria and the corresponding clicks, respectively. The model of commodity attribute $p\left(\omega \mid H_{C}\right)$ can be calculated from cumulative average of history click $\operatorname{model} p\left(\omega \mid C_{i}\right)$ : 


$$
p\left(\omega \mid H_{C}\right)=\frac{1}{k-1} \sum_{i=1}^{k-1} p\left(\omega \mid C_{i}\right)
$$

The weight of commodity attribute of history click can be achieved by TF-IDF method as:

$$
p\left(\omega \mid C_{i}\right)=T F I D F_{\omega, C_{i}}=\left(1+\log \left(t f_{\omega, C_{i}}\right)\right) \times \log \left(1+\frac{N}{d f_{\omega}}\right)
$$

where $t f_{\omega, C i}$ means the number of occurrences of commodity attribute $\omega$ in the reviews of $C_{i}$ click, $N$ is the total commodity number of search results and $d f_{\omega}$ is the number of commodity with attribute $\omega$.

Through studying all the search sessions, the commodity attributes will be achieved, which may meet all the user's demands.

4.2.1 Demand correlation. Not only the law of demand but also the influence factor of transaction can be obtained through analyzing the demand history. The frequency, quantity, level and individualization are the key parameters of transaction history. The commodities that appear in the same or near order may have demand correlation. The demand coefficient $\alpha_{i j}$ means the demand correlation between commodity $i$ and $j$, and it can be expressed as follows:

$$
\alpha_{i j}=\sum_{l=1}^{p} Q_{i j l} / \sum_{l=1}^{p} Q_{i l}
$$

Where $P$ means the total order number, $Q_{i j l}$ is the number of commodity $i$ when $j$ appears in order $l$ and $Q_{i j}$ is the number of commodity $i$ in order $l$. The range of $\alpha_{i j}$ is $[0,1]$ and $\alpha_{i j} \neq \alpha_{i j}$ usually.

By calculating the demand correlation between two demands in real time, the recommendation rule will be adjusted correspondingly.

4.2.1 Attributes co-occurrence. Co-occurrence in demand history means the phenomenon that the same or different types of attributes occur in different demands. Co-occurrence analysis is a quantitative analysis method of attributes co-occurrence in various demands, which may reveal the association among different attributes and the implied meaning of these attributes. The degree of association can be measured by the co-occurrence frequency.

For $n$ attributes, there will be one $n \times n$ co-occurrence matrix:

$$
\left(\begin{array}{ccc}
f_{11} & \cdots & f_{1 n} \\
\vdots & \ddots & \vdots \\
f_{n 1} & \cdots & f_{n n}
\end{array}\right)
$$

If the $i^{\text {th }}$ attribute and $j^{\text {th }}$ attribute occur $k$ times, the co-occurrence frequency can be expressed as:

$$
f_{i j}=k
$$

\subsection{Presentation method}

The physical, knowledge and remind information of the user can be presented thoroughly through words, images, VR, AR, RTV and real-time interaction. Then the logical 
information world and the actual physical world can be syncretized, which will enhance the reality and reliability of the subject.

The ontological structure of an individual can be presented properly from five dimensions: physiology, personality, knowledge, belief and experience. The physiological dimension can be presented by a 3D model which can be used in virtual fitting and a few power bars showing the condition of body, such as physical strength, mental state and body immunity. The radar analysis is adopted in the personality dimension, because the radar chart can show the preferences intuitively. The personalized portal knowledge dimension includes education and profession. The education shown on the map contains position, school badge, specialty and time interval. The basic information of profession can be shown by the organization chart of the company, and the relationships and experience of profession can be presented by networking and event list, respectively. The belief dimension shows the statistical analysis result of viewpoint and comment by keywords and grades.

\section{Commodity modeling}

\subsection{Data sources}

The drastic competitiveness makes the commodity life-cycles shorter and shorter; as a result, holographic commodity information is the foundation of choosing the appropriate commodity. Commodity information can be obtained mainly from the supply side and demand side.

5.1.1 Supply side. The real-time information associated with the commodity can be gathered by IOT, ERP, vehicle networking and other external data, and all the information will be stored on block chain. And the retrospective system is based on the automatically collected information.

Besides the real-time information, the supplier should provide standardly description of the commodity. Then, it is easy to recognize the accurate information, which may be helpful to search and choose the optimal commodity from the numerous commodities. From the standardized description of commodity information, the keywords and quantitative information can be obtained. A standardized description method for heterogeneous commodity should contain the following information (Table I).

5.1.2 Demand side. The demand information may be mixed with uncertain linguistic information because of the limited experience and knowledge background (Xu, 2006). The complexity of the commodity itself may also reduce the accuracy of demand information. Meanwhile, multi-granularity and multi-semantic are adopted by different consumers in describing the demand information. So, it is necessary to design a standardized description method for heterogeneous demands. A standardized description method for heterogeneous demand should be included but not be limited to the following information listed in Table II.

5.1.3 Extra information. Scattered pieces of information can be obtained from the manufactures, couriers and users, e.g. the additional information, application information and evaluation information. Extra information acquired intelligently from the transactions within one portal and among portals may satisfy potential demand based on the demand correlation, attribute co-occurrence, etc. And the accuracy of recommendation may improve.

\subsection{Characteristic analysis}

5.2.1 Studied information. The more information commodity provides to the customer, the more it helps to improve the system's matching accuracy and speed. The supply history of one commodity can reflect the business strategy of the supplier. Promotions can be inferred 


\begin{tabular}{|c|c|c|}
\hline & & \\
\hline $\begin{array}{l}12 \\
10\end{array}$ & Items & Meaning \\
\hline & Category & Physical or digital \\
\hline & Industry & A-Z \\
\hline & Brand level & $1-5$ \\
\hline & Level & $1-5$ \\
\hline & Specification & Size and packing \\
\hline 178 & Valid date & Whether need sales promotion \\
\hline & $\begin{array}{l}\text { Price index } \\
\text { Sales information }\end{array}$ & $\begin{array}{l}\text { Historical and average price, trend } \\
\text { Time, location, price, volume }\end{array}$ \\
\hline & Image & Products and environment display \\
\hline & Video & Products and environment display \\
\hline & Credit & Subject and object credit \\
\hline & Label & Based on the semantic analysis \\
\hline & Instruction & Detail information \\
\hline & Link & More information \\
\hline & Cases & Typical application \\
\hline & Distributing center & Cover range, supply capacity \\
\hline & Similar commodity & Substitutability: $1-0$ \\
\hline & Inventory & Location, volume, price \\
\hline & Production capacity & Average and peak capacity \\
\hline & Supply resources & Region, quality, capacity, price \\
\hline & Ingredients & Detail information \\
\hline & Target buyers & Gender, age, job, preference \\
\hline Table 1. & Recommended item & Correlation: $1-0$ \\
\hline The standardized & Cautions & Holographic display \\
\hline description of & Main function & Application scenarios \\
\hline commodity & Storage mode & Space, temperature, humidity \\
\hline
\end{tabular}

from the phenomenon that one commodity's price remains unchanged, while its quality and sales volume increase, or commodity's quality remains unchanged while its price decreases and sales volume increases. So, except for the regular attributes, the studied information should be presented for the consumers.

5.2.2 Membership function. Linguistic variables refer to the use of words in natural language to indicate the degree of perception of commodity, rather than the traditional numerical form. In general, language is less precise than number. So the adoption of linguistic variables provides an approximate method to describe those that are too complex or subjective.

Linguistic variables are usually represented by fuzzy mathematics - by converting linguistic variables into fuzzy numbers to convey the meaning of their values, i.e. to achieve quantification. The common membership functions include trigonometric functions, trapezoidal functions and Gauss functions. Determining the membership function is subjective and uncertain to a certain extent. It should be determined according to the specific circumstances.

5.2.3 Perceptual feature space. The attributes of one commodity vary for different consumers. The semantic differential method is an effective way to measure user's perception of one commodity. First, a series of attributes of the commodity should be obtained. For each attribute, there will be 7-11 intervals between the positive meaning and negative meaning. Second, the relationship between commodity attributes and perceptual feature should be established, the TF-IDF method can be adopted. 


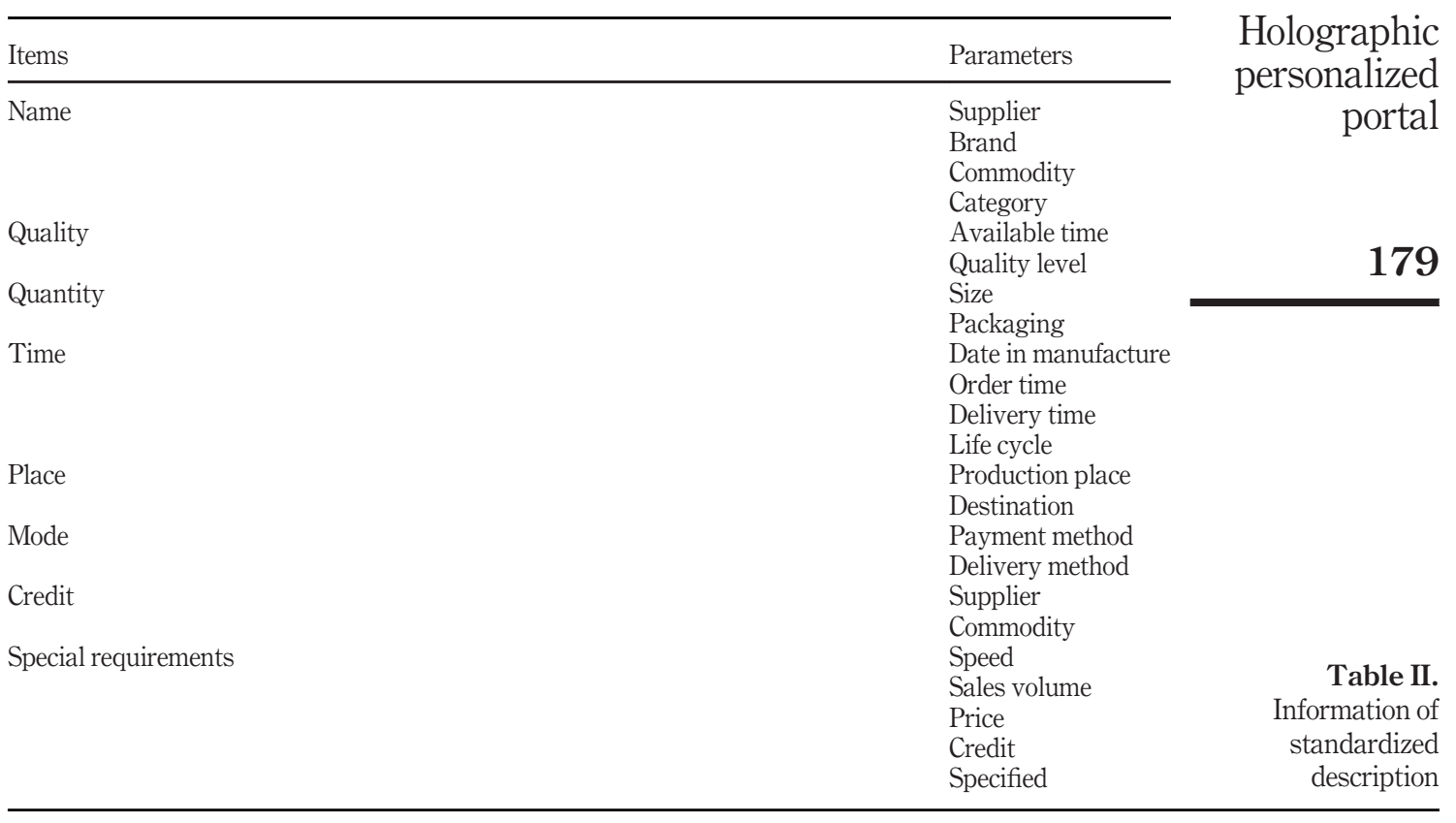

The user's attention to the product attribute can be calculated as:

$$
f_{j \rightarrow k}=n_{j \rightarrow k} / \sum_{m=1}^{c_{j}} n_{j \rightarrow m}
$$

where $n_{j \rightarrow k}$ is the number of commodity with the $k^{\text {th }}$ value of attribute $j$. Then the standard deviation of $f_{j \rightarrow k}$ should be obtained, where $c_{j}$ is the number of attribute value of attribute $j$.

$$
\mu=\frac{1}{c_{j}} \sum_{m=1}^{c_{j}} f_{j \rightarrow m}, \sigma_{j}=\sqrt{\frac{1}{c_{j}} \sum_{m=1}^{c_{j}}\left(f_{j \rightarrow m}-\mu\right)^{2}}
$$

Then the importance of each commodity attribute can be achieved as:

$$
\alpha_{j}=\frac{1-\sigma_{j}}{t-\sum \sigma_{j}}
$$

For one commodity with $t$ attributes, the importance of the commodity for the user $p$ can be expressed as:

$$
\mathrm{A}_{p}=\left(\alpha_{1}, \alpha_{2}, \cdots, \alpha_{t}\right)
$$


IJCS

1,2

180

\subsection{Presentation method}

The multi-dimensional information consists of basic information, production information, specific information, portfolio information, show information and supply and demand information. Besides the traditional images and words, VR display, voice interaction and comprehensive traceability information are adopted to display commodity (Table III).

\section{Intelligent interaction}

Based on the modeling of user and commodity, the intelligent interaction can be realized, e.g. demand decomposition, personalized search, personalized presentation, comment analysis and demand prediction.

\subsection{Demand decomposition}

The demand can be divided into explicit demand, implicit demand and unknown demand. Explicit demand includes instant demand and period demand. The implicit demand can be called predicted demand. Moreover, each kind of demand can be subdivided into noncustomized and customized demand.

The instant demand is the most common demand, and the demand information can be active input or automatic recognition by personalized portal. And the key parameters of instant demand are listed in Table II. The periodic demand is similar to the instant demand, but the demand information and the orders are sequence. In addition, there will be a configuration table to set the detailed information, such as frequency, time, duration, etc. The statistical condition of the finished transaction sequence updates constantly. The adjustment and optimization of the follow-up transaction sequence will refer to the feedback from the statistical condition and market condition. The predicted demand will be inferred or recognized based on the ontology and IOT.

\subsection{Personalized search}

There are three characteristics for personalized search:

(1) First, the user can propose demand in particular scene. As a result, not only the standardized description of demand but also the scene information will be included in the search keywords.

(2) Second, personalized information obtained from the portal, i.e. the data source of user modeling, can be the extra filter criteria. Then, there will be different search results from different users with the same search keywords.

(3) Third, personalized search can help users find what they want in the long tail. Few hot sell commodities get attention from most of the users, while few users know about the other commodities, resulting in waste of resources.

Table III.

The holographic information of commodity

Information structure

Dimensions

Basic information Production information Specific information Portfolio information Supply and demand information
Name, category, brand, bar code, specifications, date, etc.

Supplier, production situation, commodity composition, hedonic price, etc. Application scenarios, announcements, contrast situation, etc.

Related commodity, substitute commodity, composite commodity, etc.

History, statistics, status, forecast, etc. 


\subsection{Personalized presentation}

Personalized presentation will be in the user's own portal. T provide the shortest path for the user to the proper commodity, a flat and dynamic classification tag should be established. First, a new classification system should be set up to meet the needs of the dynamic mechanism. Flat classification can shorter the view path, and the present classification can selectively avoid the situation of information explosion. Second, the dynamic mechanism for classification improvement should be established. The automatic or manual controllable dynamic mechanism is based on the user interaction, e.g. searching, browsing, trading, commenting and hot-spot tracking.

\subsection{Demand prediction}

Demand prediction will reduce the risk that the system recommends the commodity which the consumer does not like anymore. The demand frequency, demand quantity, demand level and demand individualization are the key factors of demand prediction. The prediction scenario often occurs in consumables, time-expired commodities and seasonal commodities. The consumables can be shown by a Gantt chart, making it easy to know what to buy. The pervasive sensors can show the life expectancy of commodities. The seasonal commodities will be recommended in every possible scenario properly. The commodity with recommended reason will be presented by 3D models, and each commodity has its potential supply chain. Moreover, the supplier in the potential supply chain will know the potential supply chain and the demander's personalized information. Then the supplier can adjust the supply and feed the information to the demander, which may increase the transaction probability.

\section{Conclusion}

In this paper, we propose a crowd-science industrial ecological system based on a holographic personalized portal and its interaction, and takes the block-chain data storage as safeguard, which gathers the information involved in the business process. Each participant has his own holographic personalized portal, and the personalized portal consists of accurate ontology, reliable supply, intelligent demand and smart cyberspace. The personalized portal can realize the information acquisition, characteristic analysis and holographic presentation. Then, the intelligent interaction, e.g. demand decomposition, personalized search, personalized presentation and demand prediction, will be implemented within the personalized portal.

Further research can be done based in this area. First, the accuracy and instantaneity of the ontology should be improved, which is the crucial for the holographic personalized portal. Second, more accurate recommendation algorithm should be proposed for the personalized portal.

\section{References}

Adomavicius, G., Sankaranarayanan, R. and Sen, S. (2005), "Incorporating contextual information in recommender systems using a multidimensional approach", ACM Transactions on Information Systems, Vol. 23 No. 1, pp. 103-145.

Aggarwal, C.C. and Yu, P.S. (2000), "Data mining techniques for personalization”, Data Engineering Bulletin, Vol. 23, pp. 4-9.

Bruyn, A.D., Liechty, J.C. and Huizingh, E.K.R.E. (2008), "Offering online recommendations with minimum customer input through conjoint-based decision aids", Marketing Science, Vol. 27 No. 3, pp. 443-460.
Holographic personalized portal 
IJCS

1,2

182

Chuan, N.K., Sivaji, A. and Shahimin, M.M. (2013), "Kansei engineering for e-commerce sunglasses selection in Malaysia", Procedia-Social and Behavioral Sciences, Vol. 97 No. 2, pp. 707-714.

Claypool, M., Le, P. and Wased, M. (2001), "Implicit interest indicators", Proceedings of the 6th International Conference on Intelligent User Interfaces, ACM, pp. 33-40.

Joachims, T., Freitag, D. and Mitchell, T. (1997). "WebWatcher: a tour guide for the world wide web”, Proceedings of the Ijcai.

Lomax, S. and Vadera, S. (2013), "A survey of cost-sensitive decision tree induction algorithms", ACM Computing Surveys, Vol. 45 No. 2, pp. 1-35.

Koren, Y. (2009), “Collaborative filtering with temporal dynamics”, ACM, Vol. 53, pp. 447-456.

Santos, T.R.L.D. and Zárate, L.E. (2015), "Categorical data clustering: What similarity measure to recommend”, Expert Systems with Applications, Vol. 42 No. 3, pp. 1247-1260.

Smyth, B., Bradley, K. and Rafter, R. (2002), "Personalization techniques for online recruitment services", Communications of the ACM, Vol. 45 No. 5, pp. 39-40.

Williams, T., Roger, M. and Bruce, E. (2002), "Demand chain management theory: constraints and development from global aerospace supply webs”, Journal of Operations Management, Vol. 20 No. 6, pp. 691-706.

$\mathrm{Xu}, \mathrm{Z}$. (2006), "Multiple attribute decision making based on different types of linguistic information", Journal of Southeast University, Vol. 22, pp. 134-136.

Ying, X. (2003), The Research on User Modeling for Internet Personalized Services, National University of Defense Technology.

\section{About the authors}

Yadong Huang, National Engineering Laboratory for E-Commerce Technologies, Department of Automation, Tsinghua University, received his BS degree in the School of Aerospace Engineering from Beijing Institute of Technology in 2011 and master's degree in the Institute of Microelectronics from Tsinghua University in 2014. Yadong Huang's research interest includes e-commerce transaction, dynamic pricing and transaction credit evaluation.

Yueting Chai, National Engineering Laboratory for E-Commerce Technologies, Department of Automation, Tsinghua University, received his $\mathrm{PhD}$ degree in the Department of Automation from Tsinghua University in 1991 and is a Full-time Professor of the Department of Automation in Tsinghua University. Professor Chai serves as the director of National Engineering Laboratory for EBusiness Technology now with a focus on e-commerce technology and academic area. Yueting Chai is the corresponding author and can be contacted at: chaiyt@tsinghua.edu.cn

Yi Liu, National Engineering Laboratory for E-Commerce Technologies, Department of Automation, Tsinghua University, received her master's degree in the Department of Automation from Tsinghua University in 1991 and is an Associate Professor of the Department of Automation in Tsinghua University. Associate Professor Liu is now focusing on electronic commerce and supplychain management.

Xiang Gu, National Engineering Laboratory for E-Commerce Technologies, Department of Automation, Tsinghua University, received his BS degree in Department of Automation from Tsinghua University in 2016. Xiang Gu's research interest includes e-commerce transaction mode.

For instructions on how to order reprints of this article, please visit our website:

www.emeraldgrouppublishing.com/licensing/reprints.htm

Or contact us for further details: permissions@emeraldinsight.com 